\title{
The Role of Sphingomyelin Breakdown in Measles Virus Immunmodulation
}

\author{
Elita Avota Sibylle Schneider-Schaulies \\ Institute for Virology and Immunobiology, University of Wuerzburg, Wuerzburg, Germany
}

\section{Key Words}

Measles virus $•$ Immunosuppression $\cdot T$ cell silencing $•$ Dendritic cell $•$ Sphingomyelinase

\begin{abstract}
Measles virus (MV) efficiently causes generalized immunosuppression which accounts to a major extent for cases of measles-asscociated severe morbidity and mortality. MV infections alter many functions of antigen presenting cells (APC) (dendritic cells (DCs)) and lymphocytes, yet many molecular targets of the virus remain poorly defined. Cellular interactions and effector functions of DCs and lymphocytes are regulated by surface receptors. Associating with other proteins involved in cell signaling, receptors form part of receptosomes that respond to and transmit external signals through dynamic interctions with the cytoskeleton. Alterations in the composition and metabolism of membrane sphingolipids have a substantial impact on both processes. In this review we focus on the regulation of sphingomyelinase activity and ceramide release in cells exposed to $\mathrm{MV}$ and discuss the immunosuppressive role of sphingomyelin breakdown induced by MV.
\end{abstract}

Copyright $@ 2014$ S. Karger AG, Basel

\section{Introduction}

As established more than a century ago, MV efficiently suppresses especially the adaptive immune response and this almost entirely accounts for the high rates of morbidity/ mortality associated with acute measles [1]. Typically, patients succumb to secondary infections, which may follow a fatal course, and chronic or latent infections may exacerbate due to the loss of mainly $\mathrm{T}$ cell dependent immune control. Quantitative and qualitative alterations of peripheral blood lymphocytes typically occur during or for weeks after the acute infection. These include lymphopenia, an early switch from a Th1 towards a Th2 dominated response and a general inability of peripheral blood lymphocytes to expand 
in response to polyclonal or antigen-specific stimulation ex vivo $[1,2]$. The latter two characteristics mark MV as a pathogen specifically targeting cellular immunity for efficient generalised immunosuppression.

Interaction with its plasma membrane surface receptors is of major importance for measles pathogenesis also including immunosuppression $[3,4]$. Though all of them are signaling active molecules, only three receptors confer viral uptake into target cells: CD46 (used by attenuated strains in vitro [5]), nectin-4 (expressed on the basolateral side of epithelial cells, and important for viral shedding from infected hosts [6, 7]), and CD150 (also refered to as SLAMF1 [8]). In vivo, MV uptake and hematopoietic spread clearly segregate with CD150 expression patterns $[9,10]$. While MV interaction with pattern recognition receptors such as DC-SIGN and TLR2 was linked to enhancement of viral uptake into or maturation of APCs $[11,12]$, respectively, that with others such as Fc $\gamma$ RII or an as yet undefined receptor (see below) was implicated in modulating lymphocyte viability and activity in immunosuppression $[13,14]$. Within the following paragraphs we will highlight the role of stimulated sphingomyelin breakdown at the plasma membrane in two important aspects of MV induced immunomodulation: DC entry and T cell silencing.

\section{MV-DC interactions: sphingolipid breakdown mediated enhancement of infection}

The importance of the MV entry receptor, CD150, for major aspects of MV pathogenesis received direct support from studies performed in experimentally infected rhesus macaques. CD150 expression is confined to hematopoetic cells, and predominantly activated follicular B and memory Tlymphocytes are infected in secondary lymphatic tissues and, at low frequency, in PBMCs $[10,15]$. Lending support to the hypothesis that APCs in the lower respiratory tract are early, if not prime target cells for viral infection, MV infected CD150+ alveolar macrophages and CD11c+ MHC class-II+ myeloid DCs were detected in peripheral tissues early following infection $[10,16]$. This suggests that MV initially interacts with immature DCs and exploits them as Trojan horses for transport into T cell rich areas in secondary lymphatic tissues where transmission to lymphocytes occurs $[13,14,17,18]$. Unlike HIV, for which a similar scenario is postulated, MV efficiently infects DCs and this occurs in a CD150dependent manner enhanced by DC-SIGN $[12,19]$. DC-SIGN, in conjunction with TLR2, may also promote phenotypic and functional DC maturation $[11,12]$ which resembles that induced by other viruses in terms of MHC- and costimulatory molecule upregulation and cytokine release, though CCR7 upregulation and CD40 signaling may be compromised [20, 21]. To what extent these virus-induced alterations translate into the inability of MV-infected DCs to stimulate $\mathrm{T}$ cell proliferation is unknown $[13,14]$. There is, however, evidence that the majority of conjugates formed between T cells and infected DCs is unstable and does not sustain $\mathrm{Ca}^{2+}$ mobilization in T cells [22]. It appears that in addition to inadequate expression and recruitment of repulsion receptors and their ligands inhibitory signals provided by the viral glycoprotein on the DC surface to conjugating T cells are essentially involved in this process $[23,24]$, thereby rendering MV uptake for subsequent replication highly important in DC-mediated T cell silencing.

Surface expression of the MV uptake receptor CD150 is low on myeloid DCs in vitro and in vivo $[19,25]$, and membrane compartimentalization or clustering of this receptor has not yet been addressed on these cells. Lateral segregation followed by receptor concentration also including those for pathogens was, however, described following acid sphingomyelinase (ASM) activation, subsequent ceramide release and condensation of ceramide microdomains into extended ceramide enriched platforms [26, 27]. DC-SIGN is the major MV attachment receptor on DCs $[19,28]$. Its signaling involves activation of c-Raf which, in general, can be subject to ceramide mediated regulation $[29,30]$, and therefore, DC-SIGN appeared to be a likely candidate in promoting sphingomyelinase activation leading to MV receptor clustering. Indeed, DC-SIGN ligation by MV, but also specific antibodies after crosslink or mannan efficiently caused transient ASM activation and surface display of ASM and 
ceramides, and this was sensitive to EGTA, amitriptyline and DC-SIGN blocking antibodies [25]. Pharmacological or siRNA-mediated genetic ablation of ASM did not affect MV binding to DCs, however, severely impaired uptake as detected by accumulation of viral proteins after 16 hrs. This was not observed in cell lines where the uptake receptor was abundantly expressed corroborating our hypothesis that DC-SIGN mediated ASM activation regulated entry receptor availability. While lateral co-segregation of abundantly expressed CD150 and transgenic DC-SIGN was observed after mannan exposure in Raji B cells, DC-SIGN ligation caused a transient increase in CD150 surface display within minutes in DCs indicating that in these cells, ASM activation caused vertical rather than lateral CD150 recruitment [25]. It appeared that CD150 was co-transported with ASM from a common intracellular storage compartment.

There are several implications of this study that extend beyond the MV system: DC-SIGN ligation is not unique to MV. Receptor sorting upon DC-SIGN mediated ASM activation and formation of ceramide-enriched platforms on DCs might well positively, but also negatively regulate pathogen uptake. Thus, sphingomyelinase exposure caused segregation CD4 from CXCR4 and thus inhibition of fusion-mediated HIV-1 uptake, and rather fostered viral endocytosis followed by lysosomal targeting [31]. Whether this would also occur in response to ASM activation has not been tested, nor has it been assessed in viral systems whether ASM activity supports membrane fusion as revealed for phago-lysosomal fusion in macrophages infected with L. monocytogenes [32, 33]. Thus, while consequences of DC-SIGN dependent ASM activation in pathogen uptake are not MV-specific, CD150 recruitment seemingly is. Because CD150 can, however, serve as pathogen recognition receptor routing gram-negative bacteria into an endolysosomal degradative compartment in murine macrophages [34], transient availability of this receptor could be important in co-infection settings. Apart from enhancing MV uptake, ASM activation was also found to be required for DC-SIGN proximal and downstream signaling [25]. While a role of ASM in membrane proximal c-Raf and ERK activation was not surprising, inhibition of NF-kB upon TLR4 co-ligation was unexpected, because enhancement of LPS-driven NF-kB activation upon DC-SIGN co-ligation was described to occur in DCs earlier [30]. While reasons for these discrepant observations have not been unraveled as yet, ASM activation in response to DC-SIGN ligation appears to regulate gene expression in DCs especially with regard to cytokine release, which, again, would be DC-SIGN, but not MV-specific.

\section{MV-interaction with T cells: actin cytoskeletal paralysis and beyond}

Several scenarios relating to MV T cell interference were described. These include 1) loss of viability occuring due to infection and/or infection-independent apoptosis. The latter can be caused by ligation of the Fc $\gamma$ RII [35], while infection related loss may result from viral cytolysis, formation of multinucleated giant cells or destruction by virus-specific effector $\mathrm{T}$ cells in secondary lymphatic tissues [15]. Because this has been particularly revealed for the memory $\mathrm{T}$ cell population, both lymphopenia but also loss of recall responses during measles were attributed to this mechanism [15]. 2) A general cytokine imbalance was described in vitro and in vivo in that an initial Th1 rapidly shifts towards a Th2 response thereby disfavoring cellular immunity [1]. 3) Thirdly, expansion of T cells isolated from patients in response to polyclonal or antigen-specific activation is strongly impaired. The latter phenomenon has been mimicked by exposure of primary or transformed $\mathrm{T}$ cells to the MV glycoprotein complex provided by infected cells, cells transfected to express this effector complex or viral particles. T cell inhibition in this system does not involve infection or cell death, but rather a profound inability to pass beyond the $\mathrm{G}_{1}$ phase [3].

It was for several reasons that regulation of phospholipid dynamics was likely of importance in MV T cell silencing. Binding of purified MV causes condensation of detergent resistant membrane microdomains on resting $\mathrm{T}$ cells and abolishes signaling initiated from the IL-2R and TCR at a membrane proximal level in vitro and ex vivo [36, 37]. MV targets activation of the PI3K, and consequently, generation of membrane phosphatidyl-inositol- 
3,4,5-phosphate $\left(\mathrm{PIP}_{3}\right)$ and recruitment of $\mathrm{PH}$ domain containing proteins (including Akt kinase and the guanosin exchange factor Vav) [37]. Akt inhibition is crucial to MV T cell silencing, because this is efficiently abrogated upon expression of a constitutively membrane associated, active Akt kinase [36]. As they are PI3K downstream effectors, MV impaired nuclear accumulation of certain splice accessory factors thereby giving rise to production of protein isoforms translated from alternatively spliced mRNAs. These included a constitutively membrane associated active lipid phosphatase (SIP110) which on overexpression interfered with T cell expansion, most likely by constitutively depleting PIP $_{3}$ at the cell membrane [38].

As a second major target of MV T cell silencing, integrity and dynamics of cortical actin proved to be effectively disturbed (refered to as T cell paralysis) which is characterized by a failure to 1) adhere to and morphologically polarise on fibronectin (FN), 2) to recruit Vav and activate small GTPases Rac1 and Cdc42 and 3) to phosphorylate ezrin/moesin proteins and cofilin, which when dephosphorylated, severs barbed ends of actin filaments, upon TCR ligation [39]. Phenotypically, MV exposure causes a rapid collapse of actin based protrusions in $\mathrm{T}$ cells which, on stimulation, are unable to spread, form lamellopodia, and correctly organize monocentric immune synapses (ISs) with mature DCs [39]. Moreover, ISs formed between infected DCs and T cells were mostly unstable and did not support sustained $\mathrm{Ca}^{2+}$ fluxing thereby explaining why infected DCs do not promote $\mathrm{T}$ cell expansion $[22,24$, 40]. Because both membrane phospholipid metabolism and actin dynamics are essential components of IS function [41, 42], it is not surprising that a virus interfering at these levels is highly efficient at abrogating $\mathrm{T}$ cell activation.

Several lines of evidence suggested that sphingomyelinase activation and ceramide release might contribute to MV-mediated T cell silencing mainly including their interference with Akt activation, cytoskeletal remodeling and ERM protein activity (though not revealed in T cells) [43-46] as well as their ability to hamper TCR-stimulated CRAC channel activation and subsequent $\mathrm{Ca}^{2+-}$ influx $[47,48]$. Moreover, inhibition of $\mathrm{T}$ cell proliferation was reported after NSM activation upon choleratoxin binding to lipid rafts or exogenous elevation of ceramides $[49,50]$.

Indeed, MV causes surface accumulation of ceramides due to activation of both NSM and ASM (the latter being displayed at the surface), with NSM most likely accounting for ASM activation. This was related to the MV gp interaction since a recombinant MV, expressing VSV G protein instead (MGV) failed to activate sphingomyelinases [3, 51]. MVinduced sphingomyelinase activation appeared not to account for loss of stimulated $\mathrm{T}$ cell proliferation, but rather for that of membrane protrusions and efficiently interfered with FN- or $\alpha \mathrm{CD} 3 / \mathrm{CD} 28$-stimulated $\mathrm{T}$ cell polarisation, surface receptor redistribution, ezrin/ moesin phosphorylation, and directional cell motility. Bacterial sphingomyelinase or biologically active $\mathrm{C}_{16}$-ceramide caused similar effects when applied instead of MV, and thus, our observations apparently reflect a general, as yet unnoted ceramide dependent block in $\mathrm{T}$ cell cytoskeletal dynamics and thereby, activation [51]. Interestingly, ASM deficient T cells revealed an aberrant morphology as well in that they developed prominent ruffles instead of microvillar structures and were unable to polarise their cortical actin on fibronectin (Avota and Schneider-Schaulies, unpublished). These observations indicate that temporally and spatially controlled sphingomyelinase activation may be of crucial importance in cytoskeletal dynamics in T cells thereby controlling essential functions such as adherence, migration, and interaction with APCs.

\section{Outlook}

Formation of unstable ISs with MV-infected DCs which do not support sustained $\mathrm{Ca}^{2+}$ mobilization in T cells strongly suggests that MV targets their architecture and function for $\mathrm{T}$ cell silencing. Because formation of functional ISs is critical to $\mathrm{T}$ cell activation, not surprisingly pathogens other than MV also target this interaction for intervention. This has been documented for DCs infected with respiratory syncytial virus or ISs involving HIVinfected T cells [52-55]. 
How sphingomyelin breakdown/ceramide release might regulate of IS function and what would be the targets is certainly a highly interesting subject to address. Little is known about the role of sphingomyelinase activity in DC maturation and function as yet nor has it been directly assessed whether association of surface receptors important in DC function (such as for instance CD80, CD86 or CD40) with microdomains is subject to regulation in response to sphingomyelinase activation. If, as revealed for other cell types, MV gps would be located in lipid rafts in DCs as well, they might redistribute upon sphingomyelin breakdown thereby possibly affecting interaction with receptors on T cells required for transmission of activatory signals. Much less studied as for T cells, actin remodeling and protrusion of actin based extensions are also critical for the ability of DCs to promote T cell activation [56], and it would be interesting to see whether this would be positively of negatively affected upon sphingomyelinase activation. As evident from several studied, sphingomyelinase activation/ ceramide release interferes with $\mathrm{T}$ cell activation, for instance by affecting actin cytoskeletal remodeling and sustained $\mathrm{Ca}^{2+}$ mobilization $[47,48,51]$, however, targets remain as yet obscure. In line with their inhibitory function, ceramide species are virtually absent from the lipid environment of activated TCR clusters [57]. It will thus be highly interesting to directly reveal as to whether infectious agents (or compounds thereof) interfering with IS function would cause either of these effects, and if so, define microdomains targeted within the IS. This, however, will only become feasible once the experimental tools will become available to address this issue.

\section{Acknowledgement}

We thank Erich Gulbins, Essen for excellent cooperation and the Deutsche Forschungsgemeinschaft for funding our laboratory work relating to the subject of this review (SPP1267).

\section{Disclosure Statement}

We disclose any sponsorship relating to our research and conflicts of interest.

\section{References}

1 Griffin DE: Measles virus-induced suppression of immune responses. Immunol Rev 2010;236:176-189.

-2 Schneider-Schaulies S, Schneider-Schaulies J: Measles virus-induced immunosuppression. Curr Top Microbiol Immunol 2009;330:243-269.

$\rightarrow 3$ Avota E, Gassert E, Schneider-Schaulies S: Measles virus-induced immunosuppression: from effectors to mechanisms. Med Microbiol Immunol 2010;199:227-237.

4 Schneider-Schaulies J, Schneider-Schaulies S: Receptor interactions, tropism, and mechanisms involved in morbillivirus-induced immunomodulation. Adv Virus Res 2008;71:173-205.

5 Yanagi Y, Takeda M, Ohno S, Hashiguchi T: Measles virus receptors. Curr Top Microbiol Immunol 2009;329:13-30.

6 Noyce RS, Bondre DG, Ha MN, Lin LT, Sisson G, Tsao MS, Richardson CD: Tumor Cell Marker PVRL4 (Nectin 4) Is an Epithelial Cell Receptor for Measles Virus. PLoS Pathog 2011;7:e1002240.

7 Muhlebach MD, Mateo M, Sinn PL, Prufer S, Uhlig KM, Leonard VH, Navaratnarajah CK, Frenzke M, Wong XX, Sawatsky B, Ramachandran S, McCray PB Jr, Cichutek K, von Messling V, Lopez M, Cattaneo R: Adherens junction protein nectin-4 is the epithelial receptor for measles virus. Nature 2011;480:530-533.

-8 Tatsuo H, Ono N, Tanaka K, Yanagi Y: SLAM (CDw150) is a cellular receptor for measles virus. Nature 2000;406:893-897.

-9 de Swart RL: The pathogenesis of measles revisited. Pediatr Infect Dis J 2008;27:S84-88.

10 de Swart RL, Ludlow M, de Witte L, Yanagi Y, van Amerongen G, McQuaid S, Yuksel S, Geijtenbeek TB, Duprex WP, Osterhaus AD: Predominant infection of CD150+ lymphocytes and dendritic cells during measles virus infection of macaques. PLoS Pathog 2007;3:e178. 
Avota/Schneider-Schaulies: Measles Virus in Sphingomyelinase Activation

11 Bieback K, Lien E, Klagge IM, Avota E, Schneider-Schaulies J, Duprex WP, Wagner H, Kirschning CJ, Ter Meulen V, Schneider-Schaulies S: Hemagglutinin protein of wild-type measles virus activates toll-like receptor 2 signaling. J Virol 2002;76:8729-8736.

12 de Witte L, Abt M, Schneider-Schaulies S, van Kooyk Y, Geijtenbeek TB: Measles virus targets DC-SIGN to enhance dendritic cell infection. J Virol 2006;80:3477-3486.

13 Kerdiles YM, Sellin CI, Druelle J, Horvat B: Immunosuppression caused by measles virus: role of viral proteins. Rev Med Virol 2006;16:49-63.

14 Schneider-Schaulies S, Dittmer U: Silencing T cells or T-cell silencing: concepts in virus-induced immunosuppression. J Gen Virol 2006;87:1423-1438.

15 de Vries RD, McQuaid S, van Amerongen G, Yuksel S, Verburgh RJ, Osterhaus AD, Duprex WP, de Swart RL: Measles immune suppression: lessons from the macaque model. PLoS Pathog 2012;8:e1002885.

16 Lemon K, de Vries RD, Mesman AW, McQuaid S, van Amerongen G, Yuksel S, Ludlow M, Rennick LJ, Kuiken T, Rima BK, Geijtenbeek TB, Osterhaus AD, Duprex WP, de Swart RL: Early target cells of measles virus after aerosol infection of non-human primates. PLoS Pathog 2011;7:e1001263.

17 Pohl C, Shishkova J, Schneider-Schaulies S: Viruses and dendritic cells: enemy mine. Cell Microbiol 2007;9:279-289.

-18 Schneider-Schaulies S, Klagge IM, ter Meulen V: Dendritic cells and measles virus infection. Curr Top Microbiol Immunol 2003;276:77-101.

19 de Witte L, de Vries RD, van der Vlist M, Yuksel S, Litjens M, de Swart RL, Geijtenbeek TB: DC-SIGN and CD150 have distinct roles in transmission of measles virus from dendritic cells to T-lymphocytes. PLoS Pathog 2008;4:e1000049.

-20 Servet-Delprat C, Vidalain PO, Bausinger H, Manie S, Le Deist F, Azocar O, Hanau D, Fischer A, RabourdinCombe C: Measles virus induces abnormal differentiation of CD40 ligand-activated human dendritic cells. J Immunol 2000;164:1753-1760.

21 Abt M, Gassert E, Schneider-Schaulies S: Measles virus modulates chemokine release and chemotactic responses of dendritic cells. J Gen Virol 2009;90:909-914.

22 Shishkova Y, Harms H, Krohne G, Avota E, Schneider-Schaulies S: Immune synapses formed with measles virus-infected dendritic cells are unstable and fail to sustain T cell activation. Cell Microbiol 2007;9:19741986.

23 Tran-Van H, Avota E, Bortlein C, Mueller N, Schneider-Schaulies S: Measles virus modulates dendritic cell/T-cell communication at the level of plexinA1/neuropilin-1 recruitment and activity. Eur J Immunol 2011;41:151-163.

24 Klagge IM, ter Meulen V, Schneider-Schaulies S: Measles virus-induced promotion of dendritic cell maturation by soluble mediators does not overcome the immunosuppressive activity of viral glycoproteins on the cell surface. Eur J Immunol 2000;30:2741-2750.

25 Avota E, Gulbins E, Schneider-Schaulies S: DC-SIGN mediated sphingomyelinase-activation and ceramide generation is essential for enhancement of viral uptake in dendritic cells. PLoS Pathog 2011;7:e1001290.

26 Gulbins E, Dreschers S, Wilker B, Grassme H: Ceramide, membrane rafts and infections. J Mol Med 2004;82:357-363.

27 Gulbins E, Kolesnick R: Raft ceramide in molecular medicine. Oncogene 2003;22:7070-7077.

28 Koethe S, Avota E, Schneider-Schaulies S: Measles virus transmission from dendritic cells to T cells: formation of synapse-like interfaces concentrating viral and cellular components. J Virol 2012;86:97739781.

29 Yao B, Zhang Y, Delikat S, Mathias S, Basu S, Kolesnick R: Phosphorylation of Raf by ceramide-activated protein kinase. Nature 1995;378:307-310.

-30 Gringhuis SI, den Dunnen J, Litjens M, van Het Hof B, van Kooyk Y, Geijtenbeek TB: C-type lectin DC-SIGN modulates Toll-like receptor signaling via Raf-1 kinase-dependent acetylation of transcription factor NFkappaB. Immunity 2007;26:605-616.

-31 Finnegan CM, Rawat SS, Cho EH, Guiffre DL, Lockett S, Merrill AH Jr, Blumenthal R: Sphingomyelinase restricts the lateral diffusion of CD4 and inhibits human immunodeficiency virus fusion. J Virol 2007;81:5294-5304.

-32 Utermohlen 0, Herz J, Schramm M, Kronke M: Fusogenicity of membranes: the impact of acid sphingomyelinase on innate immune responses. Immunobiology 2008;213:307-314.

33 Utermohlen 0, Karow U, Lohler J, Kronke M: Severe impairment in early host defense against Listeria monocytogenes in mice deficient in acid sphingomyelinase. J Immunol 2003;170:2621-2628.

-34 Berger SB, Romero X, Ma C, Wang G, Faubion WA, Liao G, Compeer E, Keszei M, Rameh L, Wang N, Boes M, Regueiro JR, Reinecker HC, Terhorst C: SLAM is a microbial sensor that regulates bacterial phagosome functions in macrophages. Nat Immunol 2010;11:920-927. 
35 Laine D, Bourhis JM, Longhi S, Flacher M, Cassard L, Canard B, Sautes-Fridman C, Rabourdin-Combe C, Valentin H: Measles virus nucleoprotein induces cell-proliferation arrest and apoptosis through NTAIL-NR and NCORE-FcgammaRIIB1 interactions, respectively. J Gen Virol 2005;86:1771-1784.

-36 Avota E, Avots A, Niewiesk S, Kane LP, Bommhardt U, ter Meulen V, Schneider-Schaulies S: Disruption of Akt kinase activation is important for immunosuppression induced by measles virus. Nat Med 2001;7:725-731.

-37 Avota E, Muller N, Klett M, Schneider-Schaulies S: Measles virus interacts with and alters signal transduction in T-cell lipid rafts. J Virol 2004;78:9552-9559.

-38 Avota E, Harms H, Schneider-Schaulies S: Measles virus induces expression of SIP110, a constitutively membrane clustered lipid phosphatase, which inhibits T cell proliferation. Cell Microbiol 2006;8:18261839.

39 Muller N, Avota E, Schneider-Schaulies J, Harms H, Krohne G, Schneider-Schaulies S: Measles virus contact with T cells impedes cytoskeletal remodeling associated with spreading, polarization, and CD3 clustering. Traffic 2006;7:849-858.

40 Schnorr JJ, Xanthakos S, Keikavoussi P, Kampgen E, ter Meulen V, Schneider-Schaulies S: Induction of maturation of human blood dendritic cell precursors by measles virus is associated with immunosuppression. Proc Natl Acad Sci USA 1997;94:5326-5331.

41 Billadeau DD, Burkhardt JK: Regulation of cytoskeletal dynamics at the immune synapse: new stars join the actin troupe. Traffic 2006; 7:1451-1460.

42 Gombos I, Kiss E, Detre C, Laszlo G, Matko J: Cholesterol and sphingolipids as lipid organizers of the immune cells' plasma membrane: their impact on the functions of MHC molecules, effector T-lymphocytes and T-cell death. Immunol Lett 2006;104:59-69.

43 Bourbon NA, Sandirasegarane L, Kester M: Ceramide-induced inhibition of Akt is mediated through protein kinase Czeta: implications for growth arrest. J Biol Chem 2002;277:3286-3292.

44 Zeidan YH, Jenkins RW, Hannun YA: Remodeling of cellular cytoskeleton by the acid sphingomyelinase/ ceramide pathway. J Cell Biol 2008;181:335-350.

45 Hannun YA, Obeid LM: Principles of bioactive lipid signalling: lessons from sphingolipids. Nat Rev Mol Cell Biol 2008;9:139-150.

46 Canals D, Roddy P, Hannun YA: Protein phosphatase 1alpha mediates ceramide-induced ERM protein dephosphorylation: a novel mechanism independent of phosphatidylinositol 4, 5-biphosphate (PIP2) and myosin/ERM phosphatase. J Biol Chem 2012;287:10145-10155.

47 Lepple-Wienhues A, Belka C, Laun T, Jekle A, Walter B, Wieland U, Welz M, Heil L, Kun J, Busch G, Weller M, Bamberg M, Gulbins E, Lang F: Stimulation of CD95 (Fas) blocks T lymphocyte calcium channels through sphingomyelinase and sphingolipids. Proc Natl Acad Sci USA 1999;96:13795-13800.

48 Church LD, Hessler G, Goodall JE, Rider DA, Workman CJ, Vignali DA, Bacon PA, Gulbins E, Young SP: TNFR1induced sphingomyelinase activation modulates TCR signaling by impairing store-operated $\mathrm{Ca}^{2+}$ influx. J Leukoc Biol 2005;78:266-278.

49 Rouquette-Jazdanian AK, Foussat A, Lamy L, Pelassy C, Lagadec P, Breittmayer JP, Aussel C: Cholera toxin B-subunit prevents activation and proliferation of human CD4+ T cells by activation of a neutral sphingomyelinase in lipid rafts. J Immunol 2005;175:5637-5648.

50 O'Byrne D, Sansom D: Lack of costimulation by both sphingomyelinase and C2 ceramide in resting human T cells. Immunology 2000;100:225-230.

-51 Gassert E, Avota E, Harms H, Krohne G, Gulbins E, Schneider-Schaulies S: Induction of membrane ceramides: a novel strategy to interfere with T lymphocyte cytoskeletal reorganisation in viral immunosuppression. PLoS Pathog 2009;5:e1000623.

52 Gonzalez PA, Prado CE, Leiva ED, Carreno LJ, Bueno SM, Riedel CA, Kalergis AM: Respiratory syncytial virus impairs T cell activation by preventing synapse assembly with dendritic cells. Proc Natl Acad Sci USA 2008;105:14999-15004.

53 Fackler OT, Alcover A, Schwartz O: Modulation of the immunological synapse: a key to HIV-1 pathogenesis? Nat Rev Immunol 2007; 7:310-317.

54 Haller C, Rauch S, Fackler OT: HIV-1 Nef employs two distinct mechanisms to modulate Lck subcellular localization and TCR induced actin remodeling. PLoS ONE 2007;2:e1212.

55 Millington OR, Gibson VB, Rush CM, Zinselmeyer BH, Phillips RS, Garside P, Brewer JM: Malaria impairs T cell clustering and immune priming despite normal signal 1 from dendritic cells. PLoS Pathog 2007;3:1380-1387.

56 Benvenuti F, Hugues S, Walmsley M, Ruf S, Fetler L, Popoff M, Tybulewicz VL, Amigorena S: Requirement of Rac1 and Rac2 expression by mature dendritic cells for T cell priming. Science 2004;305:1150-1153.

57 Zech T, Ejsing CS, Gaus K, de Wet B, Shevchenko A, Simons K, Harder T: Accumulation of raft lipids in T-cell plasma membrane domains engaged in TCR signalling. Embo J 2009;28:466-476. 